\title{
Signal simulations in urban environments
}

\author{
A. Steingass, B. Krach, F. Schubert \\ German Aerospace Center (DLR) \\ Institute of Communications and Navigation, \\ Wessling,Germany
}

\author{
M. Crisci, R. Prieto-Cerdeira \\ European Space Agency (ESA) \\ Noordwijk \\ the Netherlands
}

\begin{abstract}
This publication explores the influence of a realistic multipath channel model to satellite navigation applications in urban environments.

In this context we have discovered that there is a tradeoff between accuracy and robustness concerning the bandwidth. While narrow band signals such as GPS C/A, BOC $(1,1)$ proved to be robust especially under shadowing situations the wideband signals lost lock very likely in the shadowing situation while being more accurate in LOS situations.
\end{abstract}

In severe multipath situations we have been able to prove the performance of the particle filter receiver wich is clearly showing a better performance than the conventional receivers and even than a maximum likelihood estimation DLL.

Furthermore we have identified critical situations where receivers tend to loose lock.

Keywords: GPS, GALILEO, Multipath, signal simulations, shadowing

\section{INTRODUCTION}

After switching off the selective availability errors caused by multipath propagation became one of the major error sources. Consequentially its mitigation became important for new satellite navigation systems. New signals and receivers have been developed but most of the have been tested in lone of sight (LOS) conditions only. In the following we will investigate the new signals and receivers in realistic propagation conditions for urban environments

\section{THE CHANNEL MODEL USED}

In 2002 we have performed extensive measurements on the multipath channel [6]. From these measurements we have developed a realistic channel model which has been published in [7] and later be standardized by ITU 681-7.

\section{RECEIVERS AND MULTIPATH MitigATION}

Multipath is today still one of the most crucial problems in GNSS, as the error is caused locally and can not be corrected through the use of correction data, which is provided by reference receiver stations or networks. The advances in the development of signal processing techniques for multipath mitigation have led to continuous improvements over the past decades, whereas basically two major approaches can be distinguished: First, the class of techniques that actually mitigate the effect of multipath by aligning the more or less traditional receiver components (see Figure 1). Most of these conventional mitigation techniques are in some way aligning the discriminator of the delay lock loop (DLL) to the signal received in the multipath environment. Well-known examples of this category are the Narrow Correlator [1] and the Strobe Correlator [2].

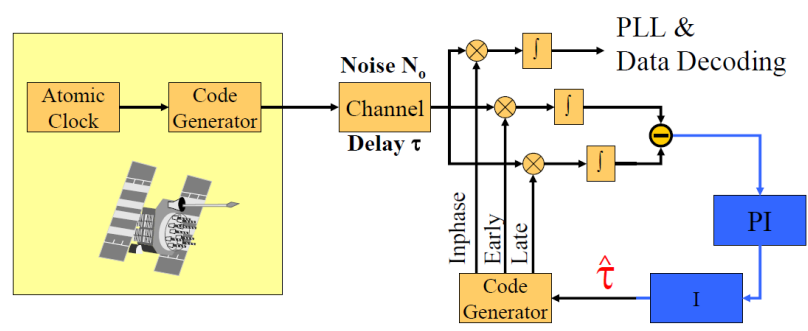

Figure 1: Signal generation, signal propagation through the channel, and signal reception with a DLL receiver.

Second, the class of multipath estimation techniques, which treat multipath, i.e. specifically the delays of the paths, as something to be estimated from the received signal, so that its effects can be trivially removed at a later processing stage. For the estimation techniques static and dynamic approaches can be distinguished, according to the underlying assumption of the channel dynamics. Examples for static multipath estimation are those belonging to the family of maximum likelihood (ML) estimators, where the probably best-known technique is the multipath estimating delay lock loop (MEDLL) [3]. During the last years sequential estimation algorithms in the form of Bayesian filters have gained some attention in the field of multipath mitigation [4]. These algorithms exploit prior knowledge about the temporal channel statistics through the use of statistical channel models, which allows one to improve the multipath performance of the receiver.

\section{A. Multipath Estimation Signal Model}

The common fundament of the estimation approaches is to consider the multipath reception explicitly when designing receiver. Hence, in a multipath estimating receiver the complex valued baseband-equivalent received signal is assumed to equal 


$$
z(t)=\sum_{i=0}^{N_{m}} a_{i} s\left(t-\tau_{i}\right)+n(t),
$$

where $s(t)$ is the transmitted navigation signal, $\mathrm{Nm}+1$ is the total number of paths reaching the receiver, and ai $(\mathrm{t})$ and $\tau \mathrm{i}(\mathrm{t})$ are their individual complex amplitudes and time delays, respectively. The signal is disturbed by additive white Gaussian noise $n(t)$ of power $\sigma 2$. Grouping blocks of $L$ samples at times $(\mathrm{n}+\mathrm{kL}) \mathrm{Ts}, \mathrm{n}=0, \ldots, \mathrm{L}-1$, together into vectors, and assuming that the delays and amplitudes are constant and equal to $\tau \mathrm{k}=(\tau \mathrm{k}, 1, \ldots, \tau \mathrm{k}, \mathrm{Nk}) \mathrm{T}$ and $\mathrm{ak}=(\mathrm{ak}, 0, \ldots, \mathrm{ak}, \mathrm{Nm}) \mathrm{T}$ within the corresponding time interval, the likelihood function for the signal parameter estimation problem is given by the complex normal distribution

$$
p\left(\mathbf{z}_{k} \mid \boldsymbol{\tau}_{k}, \mathbf{a}_{k}\right)=\mathrm{N}\left(\sum_{i=0}^{N_{m}} a_{k, i} \mathbf{s}\left(\tau_{k, i}\right) ; \sigma^{2}\right)
$$

\section{B. Maximum Likelihood Estimation}

As the naming implies the ML estimate is the set of parameters, which maximizes the likelihood function, i.e. the conditional probability of the received signal:

$$
\left\{\hat{\boldsymbol{\tau}}_{k}^{\mathrm{ML}}, \hat{\mathbf{a}}_{k}^{\mathrm{ML}}\right\}=\underset{\boldsymbol{\tau}_{k}, \mathbf{a}_{k} \mid N_{m}}{\arg \max } p\left(\mathbf{z}_{k} \mid \boldsymbol{\tau}_{k}, \mathbf{a}_{k}\right) .
$$

Since a closed form solution of (3) does not exist, several strategies have been proposed in the literature to implement the estimator, where most of them estimate the amplitudes analytically whereas the delay estimates are obtained iteratively by means of numerical optimization methods. The actual number of received paths $\mathrm{Nm}+1$, which is unknown in practice, too, is commonly estimated separately along using a statistical detection test.

For the simulations performed in this paper we solved (3) via a Newton-type method. To estimate the number of paths a likelihood ratio test was implemented.

\section{Sequential Bayesian Estimation}

In contrast to the ML estimator in a sequential Bayesian estimator the estimates are not obtained independently for each observation interval. Instead at each time step prior knowledge, which is derived from past observation intervals, is used to refine the estimates. Specifically the parameters $\tau \mathrm{k}$ and ak are estimated for each time instant $\mathrm{k}$ in terms of the a-posteriori probability density function (PDF) $\mathrm{p}(\tau \mathrm{k}, \mathrm{ak} \mid \mathrm{Zk})$, with $\mathrm{Zk}=\{\mathrm{zk}, \ldots, \mathrm{z} 0\}$ being the entire history of received measurements up to the time instant $\mathrm{k}$. The sequence of aposteriori PDFs can be computed recursively by alternating calculation of the prediction step (via the ChapmanKolmogorov equation)

$$
\begin{aligned}
& p\left(\boldsymbol{\tau}_{k}, \mathbf{a}_{k} \mid \mathbf{Z}_{k-1}\right)= \\
& \quad \int p\left(\boldsymbol{\tau}_{k}, \mathbf{a}_{k} \mid \boldsymbol{\tau}_{k-1}, \mathbf{a}_{k-1}\right) \\
& \quad p\left(\boldsymbol{\tau}_{k-1}, \mathbf{a}_{k-1} \mid \mathbf{Z}_{k-1}\right) \mathrm{d} \boldsymbol{\tau}_{k-1} \mathrm{~d} \mathbf{a}_{k-1}
\end{aligned}
$$

which exploits the statistical dependencies between successive observation intervals through the transition density $\mathrm{p}(\tau \mathrm{k}, \mathrm{ak} \mid \tau \mathrm{k}-1, \mathrm{ak}-1)$ in order to compute the a-priori PDF, and the computation of the update step

$$
p\left(\boldsymbol{\tau}_{k}, \mathbf{a}_{k} \mid \mathbf{Z}_{k}\right)=\frac{p\left(\mathbf{z}_{k} \mid \boldsymbol{\tau}_{k}, \mathbf{a}_{k}\right) p\left(\boldsymbol{\tau}_{k}, \mathbf{a}_{k} \mid \mathbf{Z}_{k-1}\right)}{p\left(\mathbf{z}_{k} \mid \mathbf{Z}_{k-1}\right)}
$$

in which the likelihood function is joined with the previous a-priori PDF. Once this a-posteriori PDF is evaluated, either the channel configuration that maximizes it can be determined - the so-called maximum a-posteriori (MAP) estimate; or the expectation can be chosen - equivalent to the minimum mean square error (MMSE) estimate:

$$
\hat{\boldsymbol{\tau}}_{k}^{\mathrm{MMSE}}=\int \boldsymbol{\tau}_{k} p\left(\boldsymbol{\tau}_{k}, \mathbf{a}_{k} \mid \mathbf{Z}_{k}\right) \mathrm{d} \boldsymbol{\tau}_{k} \mathrm{~d} \mathbf{a}_{k} .
$$

To implement the sequential Bayesian estimator we employed a marginalized particle filter, in which also the number of received paths was detected and estimated simultaneously. Details on the filter algorithm can be found in [4].

\section{EVALUATION OF RECEIVERS}

To keep focused on the native behavior of delay locked loops we used a very native realization for those receivers. Neither a lock detector nor a re-acquisition functionality have been enabled for the simulations. In other words if a receiver looses lock it will stay in this condition to the end of the simulation segment.

A statistical performance evaluation for the assessed receiver types in terms of the cumulative probability density function of the tracking errors is given in Figure 2. Both conventional DLL receivers perform quite similar, however, the $\Delta \Delta$-DLL is slightly superior in the range of errors below 20 $\mathrm{m}$. This fact indicates that the improved multipath mitigation capabilities of a $\Delta \Delta$-DLL compared to a NC-DLL are indeed exploited, given the DLL is locked properly. Interestingly, the ML-DLL performs worse than the conventional DLLs for smaller errors, but is superior for larger errors. The poor performance in the range of smaller errors is due to the limited observation time in such fast varying multipath channels. Hence, at each time instant independent estimates are obtained based on short observations of the channel and no use is made of the channel's temporal correlation. The particle receiver overcomes this limitation: Though the channel is varying fast its temporal correlation is exploited by the massive parallel estimation approach of the particle filter, which adaptively detects and tracks multipath replica and thus is able to reduce 
the multipath induced tracking errors significantly (see Figure 2).

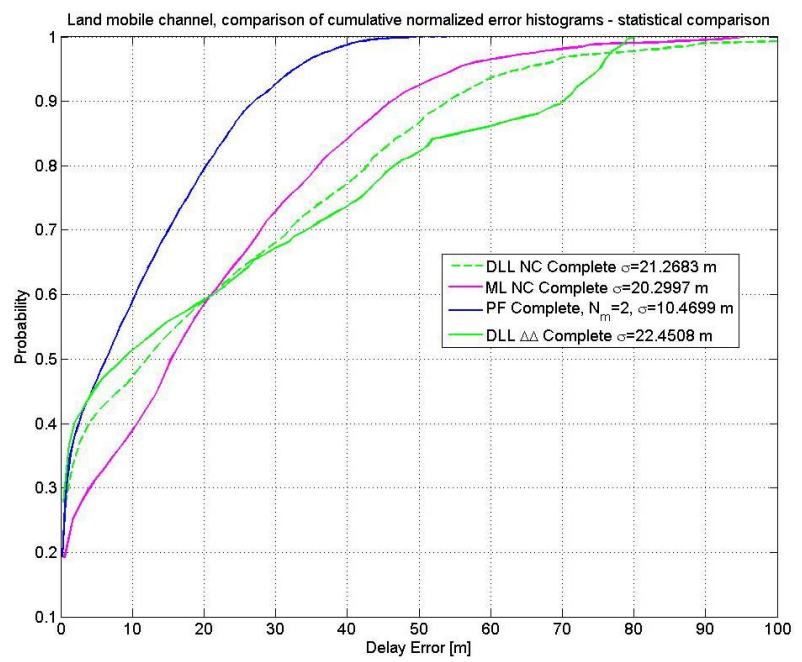

Figure 2: Direct comparison of different receivers using a GPS C/A signal.

\section{The Multipath ChanNEL'S IMPACT ON TRACKING OF DIFFERENT SIGNALS}

Four different GNSS signal types were simulated: Two of them in the L1 frequency band and two of them in Galileo's E5 band. Specifically, the simulations were run with the GPS C/A code signal (BPSK(1)), the Galileo $\operatorname{BOC}(1,1)$ signal, the AltBOC $(10,10)$ signal using its full bandwidth, and a BPSK(10) signal which uses the E5b frequency band.

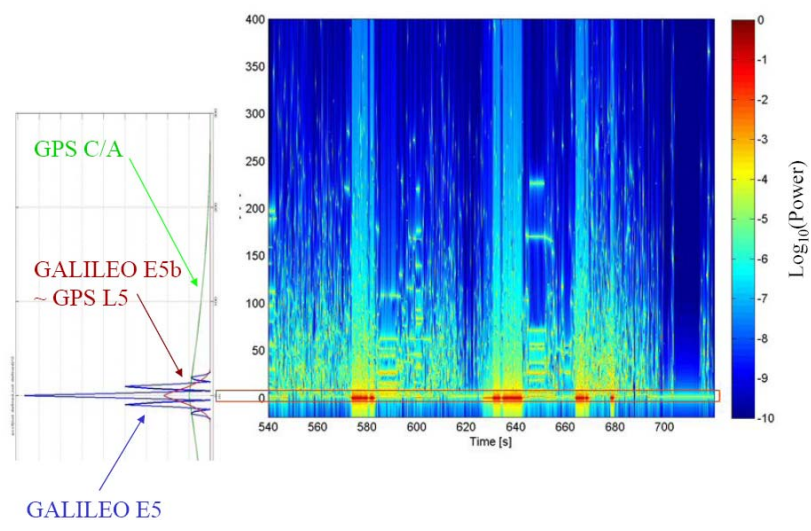

Figure 3: The simulated channel impulse response as generated by the DLR land-mobile urban channel model.

The channel impulse response which was used for the simulations is reported in Figure 3. This channel represents a challenging situation: long periods with only little power alternate with short periods of a strong line-of-sight component. The delay of the line-of-sight signal has been moved to $\tau=0$ s for every impulse response. The receiver is actually moving towards the transmitter, which can be seen as decreasing delay in the plots with the tracking results for the four respective signals, Figure 4.

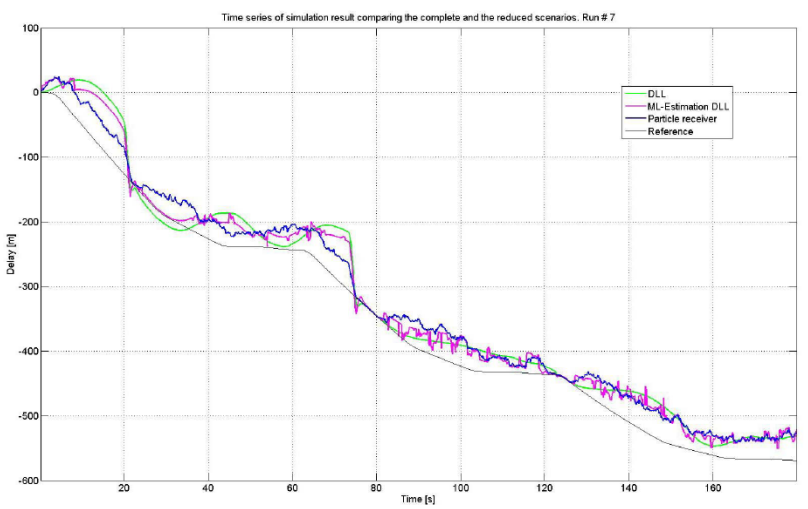

Figure 4: Pseudorange estimation for GPS C/A code signal tracking using the three employed receiver algorithms.

The $\mathrm{C} / \mathrm{A}$ code signal and the $\mathrm{BOC}(1,1)$ signal tracking meets the challenge of the difficult channel conditions. Although, of course, the navigation error is increased in periods where the LOS is shadowed the receiver is able to track the signal robustly.

Yet it turned out that the wideband signals lost lock very often than the narrowband signals. The loss of lock happens usually in a situation where the LOS is obstructed. Assuming that the DLL is perfectly synchronized, the correlators are acting as a filter with the impulse response being the signal in space itself. Result of filtering a GNSS signal with this filter would be its autocorrelation function.

We plotted the autocorrelation functions of the different signals in Figure 3 in the same scale as the channel impulse response. The trivial fact that a higher chip rate leads to a narrower autocorrelation function shall be mentioned. This results in the capability of the wideband signal to suppress multipath reflections better than the narrow band signals.

According to [5] the output of a correlator at time $t$ with lag $\tau$ can be written as

$$
C(t, \tau)=\frac{1}{T_{c}} \sum_{i=0}^{N} a_{i}(t) \cdot \varphi_{s s}\left(\tau-\tau_{i}(t)\right)
$$

if the channel parameters are assumed to be constant within the correlation interval. The number of multipath components is denoted by $\mathrm{N}$, the complex weight of each component is given by ai $(\mathrm{t})$ and their delay is given by $\tau i(\mathrm{t})$. The signal's autocorrelation function is given by $\varphi s s(t)$. If the autocorrelation function of a specific signal $\varphi s s(\tau 1)=0$ for a given multipath component delay $\tau 1(\mathrm{t} 1)$, this component is filtered out and does not contribute to the correlation result anymore.

This explains why a wideband signal has the - intended capability to suppress near echoes much better than its narrowband counterparts. 
This advantage of filtering of multipath reception in situations where the LOS is present is obvious but converts into a disadvantage where the LOS is shadowed. Now the correlators are filtering out all the echoes. Additionally, since the LOS is no longer present the DLL is losing lock caused by a lack of signal power.

Narrowband signals still gather the power provided by multipath components with larger delay. In Figure 3 the red box shows the part of the channel being used by the E5 signals main lobe. The picture illustrates very well the amount of multipath energy that is filtered out by the E5 signal in contrary to the GPS C/A code signal.

This tradeoff between robustness and accuracy seems to be valid independently of the signal itself: Wideband signals are in general more accurate in LOS conditions but are less robust in obstructed situations. The narrow band signal is more robust in obstructed situations since it can use the power of the reflections for tracking but its accuracy is in general lower.

\section{A. Direct comparison of $G P S C / A$ vs. $B O C(1,1)$}

The direct comparison between GPS C/A and GALILEO BOC $(1,1)$ is shown in Figure 5 . It can clearly be seen that for any receiver being simulated the use of $\mathrm{BOC}(1,1)$ results in more accurate results than for GPS C/A. It shall be mentioned that also the $\operatorname{BOC}(1,1)$ is less robust than the GPS C/A signal. But due to the small difference in bandwidth this reduced robustness ins only minor. The $\mathrm{BOC}(1,1)$ is robust enough to keep synchronized in most of the shadowed situations.

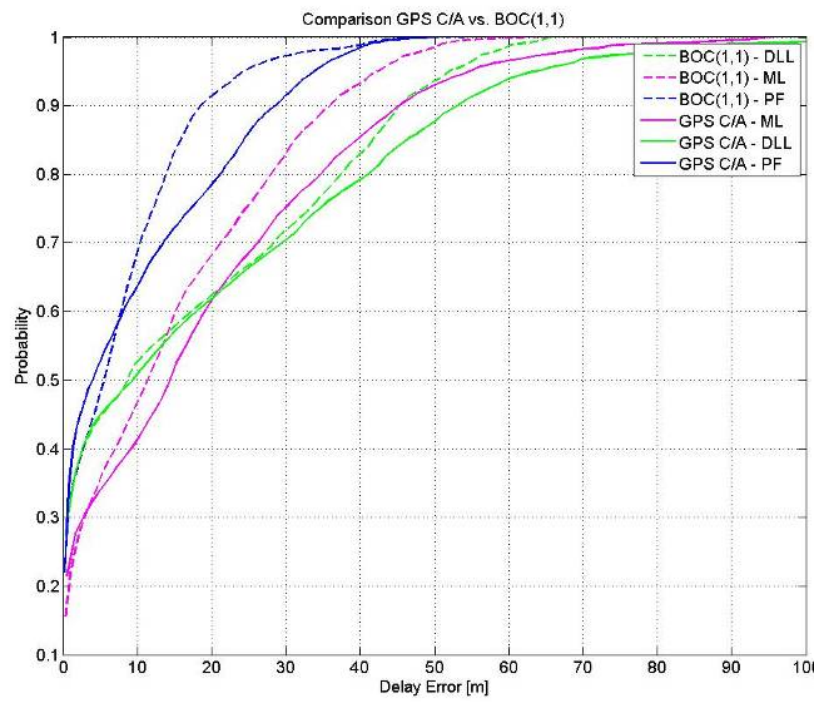

Figure 5: Direct comparison between GPS C/A and BOC $(1,1)$

\section{CRITICAL RECEIVER SITUATIONS}

During this activity we have identified critical situations for DLL based receivers.

Figure 6 is showing the channel impulse responses during this event. It is peculiar that at the moment of loosing lock the channel state changes severely. During nearly the whole segment the channel is shadowed but only during the seconds 73-75 and $85-87$ the line of sight comes through for a short moment. We name this phenomenon "Short line of sight hit" or SLOSH for short. To understand the reaction of the receiver Figure 1 shows its structure. As being a classical DLL the early-late detector is realized as a standard narrow correlator. It shall be mentioned that the detector output is not divided by the signal amplitude to prevent zero forcing. The main correction elements are realized in two integrators one as a PI-element and one as an integrator. While the LOS is shadowed the receiver get low correction outputs from the detector. It can track the signal with a quite high but for the given channel conditions reasonable error during the shadowing period. Then at second 73 the LOS hits the receiver and suddenly the E/Ldetector is generating a big output. If the LOS would stay present over a longer period the receiver would be set properly on the correct delay. But since the LOS disappears quickly after two seconds the DLL is not in a steady stay. Unfortunately the big output during the SLOSH has been fed into the integrators and has been accumulated there. Now the correction output of these integrators is mainly driven by the input during the SLOSH since the correction signal after it is again very weak. This behavior can clearly be seen in Figure 7. from second 75 the estimated delay is deviating constantly from the correct value. Now in second another SLOSH hits the receiver. Again when the LOS reappears the detector output is huge since the offset is large and the correlator outputs are big. The DLL tries immediately to correct its estimate to the corrected value. And again before reaching a steady state the LOS disappears. And again the receiver is executing the last command stored in the integrators since again the detector output is weak. As a final result the DLL is loosing lock quickly. This example shows how important it is to careful design loss of lock indicators which are adapted to shadowing situations to declare loss of lock quickly which enables a fast re-acquisition.

We consider the SLOSH as a critical situation for a DLL especially if it occurs as a double feature. The "flywheel effect" of the integrators are misleading the DLL severely after the LOS disappears. Interestingly this effect is not happening for a particle receiver since this receiver judges its estimates directly with the current measurements. There are no integrators necessary in this concept that could mislead the receiver. 


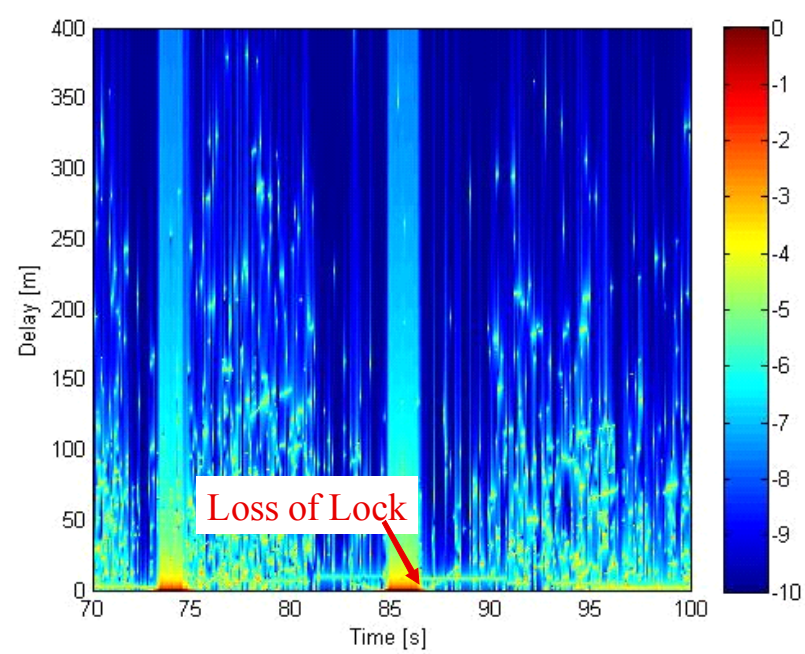

Figure 6: Channel impulse response plot for the loss of lock situation.
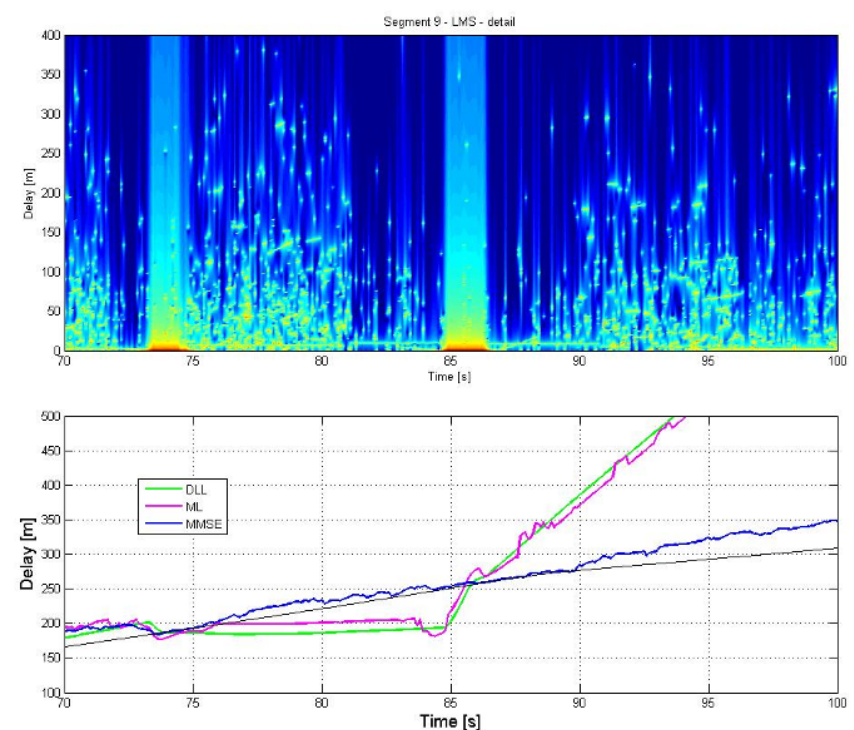

Figure 7: The loss of lock situation in detail.

\section{ACKNOWLEDGMENTS}

The work reported in this paper has been performed under ESA contract 21853/2008/F/WE. The views presented in the paper represent solely the opinion of the authors and not necessarily the view of ESA.

\section{REFERENCES}

[1] A. J van Dierendonck, P. Fenton, T. Ford, "Theory and Performance of Narrow Correlator Spacing in a GPS Receiver," Journal of The Institute of Navigation, Vol. 39, No.3 Fall 1992

[2] L. Garin, F. van Diggelen, and J. Rousseau, "Strobe and Edge correlator multipath mitigation for code," in Proceedings of the 9th International Technical Meeting of the Satellite Division of the Institute of Navigation (ION GPS 96), Kansas City, Missouri, USA, 1996, pp. 657-664.

[3] R. D. J. van Nee, J. Siereveld, P. C. Fenton, and B. R. Townsend, "The Multipath estimating delay lock loop: approaching theoretical accuracy limits," in Proceedings of the IEEE Position Location and Navigation Symposium (PLANS 94), Las Vegas, Nevada, USA, Apr. 1994, pp. 246-251

[4] M. Lentmaier, B. Krach, P. Robertson, T. Thiasiriphet, "Dynamic Multipath Estimation by Sequential Monte Carlo Methods," in Proceedings of the 20th International Technical Meeting of the Institute of Navigation Satellite Division (ION GNSS 2007), Fort Worth, Texas, USA, Sept. 2007, pp. 1712-1721.

[5] Frank M. Schubert, Thomas Jost, Patrick Robertson, Roberto PrietoCerdeira, and Bernard H. Fleury: Evaluating Tracking Performance And A New Carrier-to-Noise Estimation Method Using SNACS, Position Location and Navigation System Conference (PLANS 2010), Palm Springs, California, USA

[6] Steingass A., Lehner A.: "Measuring the navigation multipath channel a statistical analysis", ION GPS 2004 Conference Long Beach, California USA, September 2004.

[7] A. Steingaß, A. Lehner, "A channel model for land mobile satellite navigation", European Navigation Conference ENC-GNSS 2005, Munich, (2005). 